\title{
Perfil de pacientes submetidos à cirurgia de revascularização miocárdica: implicações para o cuidado de enfermagem ${ }^{1}$
}

\section{Profile of patients undergoing to coronary artery bypass grafting: implications for nursing care}

\author{
Perfil de pacientes sometidos a injerto de derivación coronaria: \\ implicaciones en el cuidado de enfermería
}

Michelle Villas Boas Fernandes ${ }^{\mathrm{I}}$, Graziella AlitiI ${ }^{\mathrm{II}}$, Emiliane N. SouzaIII

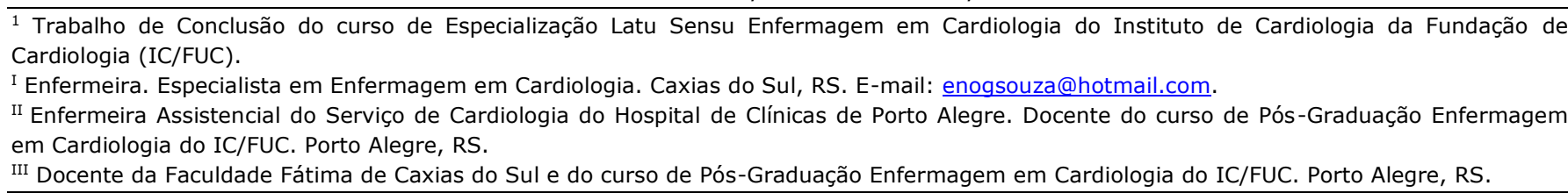

\section{RESUMO}

A gravidade dos pacientes submetidos à cirurgia de revascularização do miocárdio (RM) tem aumentado consideravelmente nas últimas décadas. O objetivo deste estudo de coorte contemporâneo foi identificar o perfil clínico e cirúrgico dos pacientes submetidos à RM. Os dados foram extraídos do prontuário de 58 pacientes internados num hospital de referência em cardiologia de Porto Alegre, Rio Grande do Sul, no ano de 2006, desde a sua chegada até a alta ou o 70 dia de permanência na unidade de terapia intensiva (UTI). A idade média foi $65,3 \pm 9,7$ anos, predominância do sexo masculino (70,7\%), hipertensão arterial sistêmica foi a comorbidade mais prevalente (86,2\%), seguida por dislipidemia (60,3\%) e diabetes (36,2\%). A cirurgia de RM isolada foi realizada em $82,8 \%$ da amostra, com circulação extracorpórea em $39,7 \%$ das cirurgias. Complicações pós-operatórias ocorreram em 48,3\% dos pacientes; a fibrilação atrial (FA) foi a complicação mais frequente $(20,7 \%)$. Houve associação entre idade, tempo de permanência na UTI e complicações pós-operatórias ( $p<0,05)$. Os pacientes submetidos à RM são majoritariamente homens, idosos, com fatores de risco clássicos para cardiopatia isquêmica, sendo a FA a complicação mais prevalente. O conhecimento acerca do perfil dos pacientes operados proporciona subsídios para a enfermagem planejar o cuidado em UTI.

Descritores: Perfil de saúde; Revascularização miocárdica; Complicações pós-operatórias; Cuidados de enfermagem.

\section{ABSTRACT}

The severity of patients undergoing coronary artery bypass grafting (CABG) has increased considerably in recent decades. This contemporaneous cohort study aimed to evaluate the clinical and surgical profile of patients undergoing CABG. Data were retrieved from medical records of 58 inpatients in hospital of reference in cardiology in Porto Alegre - Rio Grande do Sul, in the year of 2006, from the day of hospitalization until discharge, or during the 7 first days of stay in the intensive care unit (ICU).Patients were mostly males (70.7\%) with a mean age $65.3 \pm 9.7$ years; the most prevalent comorbidities were systemic arterial hypertension $(86.2 \%)$, dyslipidemia $(60.3 \%)$ and diabetes $(36.2 \%)$. Forty-eight patients $(82.8 \%)$ were submitted to CABG, with extracorporeal circulation in $39.7 \%$ of the cases. Postoperative complications, represented mostly by atrial fibrillation (AF) (20.7\%), were observed in $48.3 \%$ of the patients. Correlations were observed between age, length of stay in the ICU and incidence of postoperative complications $(p<0.05)$. Most patients undergoing CABG were male, aged and with classical risk factors for ischemic heart disease, among which AF is the most prevalent complication. The characteristics of patients undergoing myocardial revascularization surgery are of great importance for the nursing team to plan and deliver best quality care to ICU patients.

Descriptors: Health profile; Myocardial revascularization; Postoperative complications; Nursing care.

\section{RESUMEN}

La gravedad de los pacientes sometidos a injerto de derivación coronaria (IDAC) ha aumentado considerablemente en las últimas décadas. El objetivo de este estudio (cohorte) fue identificar el perfil clínico y quirúrgico de pacientes sometidos a IDAC. Los datos fueron extraídos del prontuario hospitalar de 58 pacientes hospitalizados en un hospital de referencia en cardiología en Porto Alegre - Rio Grande do Sul, en el año 2006, desde su llegada hasta el día del alta o $7^{\circ}$ día de permanencia en la unidad de terapia intensiva (UTI). La media de edad fue de $65,3 \pm 9,7$ años, con predominio masculino $(70,7 \%)$, hipertensión arterial sistémica fue la comorbilidad más prevalente $(86,2 \%)$, seguida por dislipidemia (60,3\%) y diabetes (36,2\%). La cirugía RM aislada fue realizada en $82,8 \%$ del grupo. Complicaciones post operatorias ocurrieron en $48,3 \%$ del grupo; fibrilación arterial (FA) fue la complicación más frecuente (20,7\%). Hubo asociación entre edad, tiempo de permanencia en UTI y complicaciones post operatorias $(p<0.05)$. Los pacientes sometidos a IDAC son mayoritariamente hombres, de edad avanzada, con factores de riesgo clásicos para cardiopatía isquemica, siendo FA la complicación más prevalente. El conocimiento acerca del perfil de los pacientes operados facilitaría el cuidado en la UTI proporcionado por el equipo de enfermería.

Descriptores: Perfil de salud; Revascularización miocárdica; Complicaciones postoperatorias; Atención de enfermería. 


\section{INTRODUÇÃO}

A terapêutica de portadores de cardiopatia isquêmica vem sofrendo modificações nos últimos anos, havendo significativos avanços clínicos, percutâneos e cirúrgicos. A revascularização do miocárdio (RM) é uma das opções no tratamento desses indivíduos, tendo como objetivo o aumento da sobrevida, o alívio da dor anginosa, a proteção do miocárdio isquêmico, a melhora da função ventricular, a prevenção de novo infarto agudo do miocárdio (IAM) e a recuperação física, psíquica e social do paciente, melhorando sua qualidade de $\operatorname{vida}^{(1-3)}$.

A gravidade dos pacientes submetidos à cirurgia de RM tem aumentado consideravelmente nas últimas décadas, trazendo, como consequência, o aumento dos riscos de complicações trans e pósoperatórias $^{(3-4)}$. Muitos pesquisadores têm verificado - aumento na idade de candidatos à cirurgia cardíaca, a presença de maior número de mulheres e indicação de cirurgia frente a manifestações clínicas de angina menos grave. Somando-se a isso, encontra-se uma maior incidência de IAM recente, mais disfunção ventricular esquerda, aumento do número de re-operações, taxas mais altas de candidatos à cirurgia com doença multi-arterial e com maior número de fatores condicionantes, tais como hipertensão arterial, diabetes mellitus, dislipidemia, arritmias e insuficiência cardíaca ${ }^{(5)}$. Além desses fatores, a idade, o sexo feminino, RM prévia, presença de comorbidade como doença pulmonar obstrutiva crônica, insuficiência renal e doença vascular periférica constituem condições especiais para a indicação cirúrgica(3).

De fato, o perfil do paciente com cardiopatia isquêmica tem se modificado ao longo dos anos, uma vez que os pacientes apresentam-se com mais fatores de risco cardiovasculares associados, tornando-os mais graves para enfrentarem os procedimentos terapêuticos, assim como suscetíveis às complicações. Dentre as complicações pósoperatórias estão o IAM, a síndrome do baixo débito, arritmias, disfunções pulmonares, insuficiência renal, acidentes vasculares cerebrais e infecções(3). Pacientes que apresentaram uma ou mais complicações têm mortalidade pós-operatória entre 8 a $10 \%$ maior do que aqueles que não as desenvolveram ${ }^{(2)}$. A mortalidade hospitalar no período pós-operatório tem variado de 1 a $10 \%{ }^{(5)}$.

A adequada avaliação no pré-operatório, por meio da identificação de fatores de risco cirúrgico e do desenvolvimento de medidas capazes de minimizá-los ou neutralizá-los, tem se refletido na redução da morbimortalidade operatória( ${ }^{(6)}$. Nesse contexto, a completa avaliação de enfermagem, baseada na identificação dos sinais e sintomas do período pré-operatório e na história de saúde pregressa, pode sinalizar o comportamento pósoperatório dos pacientes submetidos à RM. A partir desse conhecimento, a formulação dos diagnósticos de enfermagem e do planejamento individualizado dos cuidados, guia o enfermeiro no desenvolvimento do raciocínio clínico para prever e prover os cuidados necessários a cada situação(7).

Toda a equipe multiprofissional, em especial a enfermagem, tem papel fundamental na recuperação da saúde e bem-estar dos pacientes submetidos à cirurgia de RM. Para alcançar melhores resultados no pós-operatório, em unidade de terapia intensiva (UTI), uma adequada assistência da equipe de profissionais de enfermagem deve ser capaz de evitar ou minimizar possíveis complicações numa população potencialmente mais grave, visando também à redução do tempo de permanência na UTI e, consequentemente, à diminuição considerável nos custos. Para tanto, torna-se necessário conhecer o perfil dos pacientes, bem como as complicações mais incidentes no período pós-operatório, a fim de levantar subsídios para o preparo e qualificação da equipe de enfermagem frente às demandas de cuidado.

Assim, este estudo teve por objetivo identificar o perfil clínico e cirúrgico dos pacientes submetidos à cirurgia de RM em um hospital de referência em cardiologia, bem como as complicações pósoperatórias.

\section{MÉTODOS}

Estudo com delineamento de coorte contemporâneo, realizado na UTI de um hospital de referência em cardiologia de Porto Alegre, Rio Grande do Sul (RS). Essa UTI possui um total de 20 leitos, distribuídos da seguinte forma: 10 leitos destinados a pacientes cardiológicos clínicos (Unidade Coronariana) e 10 leitos destinados ao atendimento de pacientes pós-operatórios (Unidade Cirúrgica). Possui taxa de ocupação de $88 \%$ e média de permanência de 3,9 $\operatorname{dias}^{(8)}$.

Participaram deste estudo todos os pacientes adultos, de ambos os sexos, com idade igual ou superior a 18 anos, submetidos à cirurgia de RM eletiva, no período de março a junho de 2006, que estiveram internados na UTI do referido hospital. Pacientes submetidos à cirurgia de urgência ou emergência foram excluídos.

As variáveis demográficas foram sexo, idade e raça. As variáveis clínicas foram índice de massa corporal, fração de ejeção ventricular, comorbidades como hipertensão arterial sistêmica (HAS), dislipidemia, diabetes mellitus, tabagismo, histórico familiar de cardiopatia isquêmica, doença pulmonar obstrutiva crônica, eventos prévios como infarto agudo do miocárdio (IAM) e RM cirúrgica ou percutânea prévia, doença cérebro-vascular, 
insuficiência renal e vasculopatia periférica. As variáveis cirúrgicas estudadas foram: tipo de cirurgia, utilização e tempo de circulação extracorpórea, número de vasos lesados e enxertos, tempo de permanência na UTI e complicações pós-operatórias durante a estada nessa unidade.

As complicações pós-operatórias consideradas

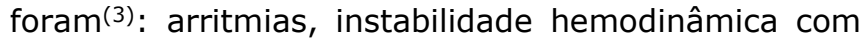
sinais de baixo débito, infarto agudo do miocárdio, ventilação mecânica prolongada (> 24 horas), acidente vascular encefálico, insuficiência renal, reoperação, parada cardiorrespiratória e derrame pleural, desde que registradas por médico intensivista no prontuário do paciente.

Para coleta dos dados foi utilizado um formulário elaborado especificamente para este fim, dividido em quatro partes: dados de identificação, dados da cirurgia, dados pré-operatórios e complicações pósoperatórias. O preenchimento do formulário foi realizado com base na revisão das informações contidas em prontuário, a partir do momento de chegada do paciente à UTI até o que ocorresse primeiro, a sua alta ou o 70 dia de permanência na UTI. A aplicação do mesmo foi realizada pela pesquisadora. O estudo foi aprovado pelo Comitê de Ética em Pesquisa da instituição hospitalar pesquisada, sob o número 101/06. Em se tratando de estudo com pesquisa em prontuário, não se previu qualquer risco de dano aos pacientes; foi observado o caráter sigiloso do manuseio de informações médicas, assinando-se um termo de responsabilidade para a utilização de prontuários.

Os dados foram analisados com o auxílio do pacote estatístico SPSS 13.0. As variáveis contínuas foram apresentadas com média e desvio padrão ou mediana e percentis interquartis, conforme apresentassem ou não distribuição normal. Os dados categóricos foram apresentados com frequências absolutas e relativas. Para comparação das variáveis contínuas entre grupos, foi utilizado o teste $t$ de Student ou Mann-Whitney, conforme os dados seguissem ou não pressupostos paramétricos. Para comparação entre as variáveis categóricas, foi utilizado o teste Exato de Fisher e Qui-quadrado. Foram considerados significativos valores de $p<0,05$.

\section{RESULTADOS E DISCUSSÃo}

A amostra foi composta por 58 pacientes, predominantemente do sexo masculino $(70,7 \%)$, com média de idade de 65,3土9,7 anos, variando de 43 a 86 anos, procedentes, na sua maioria, de Porto Alegre e região metropolitana $(63,8 \%)$, sendo $95,7 \%$ caucasianos. A média do índice de massa corpórea foi de 28,9 $\pm 4,1$, indicando sobrepeso. Todos os pacientes do estudo apresentavam pelo menos um fator de risco para cardiopatia isquêmica, sendo a hipertensão arterial sistêmica (HAS) o mais prevalente $(86,2 \%)$ seguido pela dislipidemia $(60,3 \%)$, diabete mellitus $(36,2 \%)$, tabagismo $(27,6 \%)$ e história familiar para doença isquêmica $(20,7 \%)$. Dentre os pacientes da amostra, 34,2\% já haviam sofrido IAM previamente e 15,5\% já haviam sido submetidos à RM. A fração de ejeção do ventrículo esquerdo (FE) média foi de $55,7 \pm 15,8 \%$, indicando que está no limite inferior de normalidade(3). As características da amostra estão sumarizadas na Tabela 1.

Tabela 1: Características demográficas e clínicas da amostra $(n=58)$. Porto Alegre-RS, 2006.

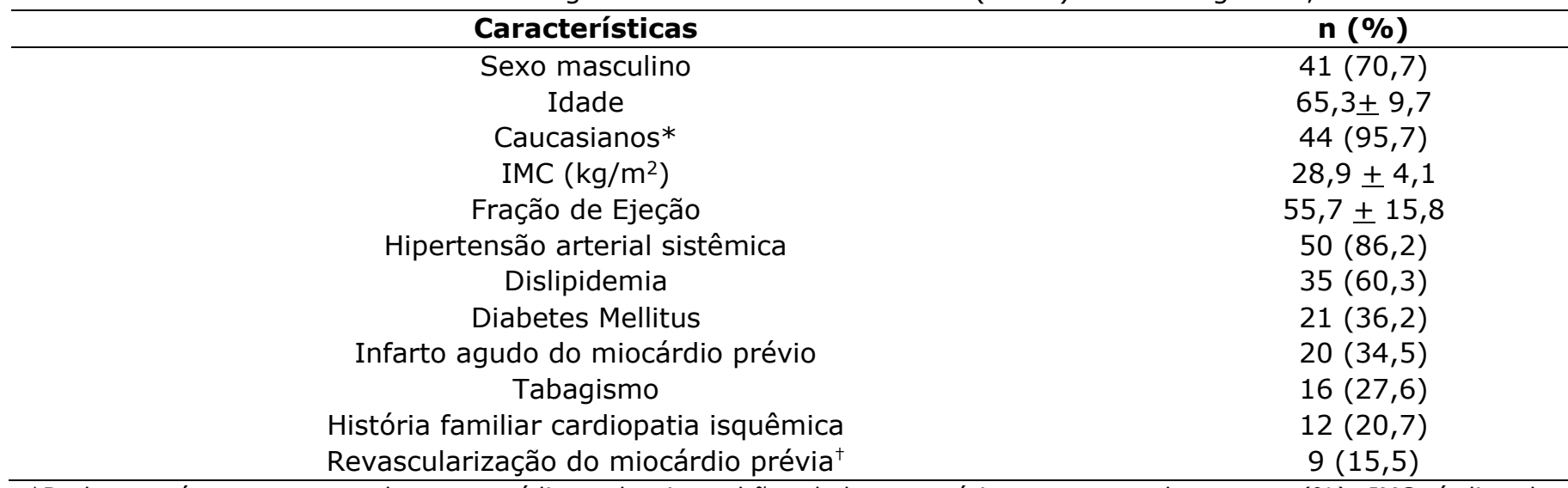

*Dados contínuos apresentados com média \pm desvio padrão; dados categóricos apresentados com n (\%). IMC: índice de massa corporal. ${ }^{*} \mathrm{n}=46$, pois nem todos os pacientes tinham registrado o dado em prontuário. ${ }^{\dagger}$ Cirúrgica ou percutânea.

Os dados deste estudo mostram que a maioria dos pacientes submetidos à RM, em nosso centro, é constituída por homens, idosos, com sobrepeso e fatores de risco clássicos para a cardiopatia isquêmica, como HAS, diabetes e dislipidemia. A predominância do sexo masculino, fato esperado, corrobora os achados de estudos, os quais relatam que as mulheres são menos submetidas à revascularização cirúrgica do miocárdio que os homens, tendência essa que vem se modificando atualmente, pois o benefício cirúrgico é igual em ambos os $\operatorname{sexos}^{(3)}$. 
Um recente estudo comparou o perfil clínico e cirúrgico entre dois grupos de pacientes submetidos à RM com intervalo de 10 anos $^{(6)}$. Os dados mostraram que pacientes atualmente submetidos à cirurgia de RM são mais idosos e em piores condições clínicas (cardíaca e sistêmica) que os operados há mais de 10 anos. A média de idade de todos os pacientes tem aumentado progressivamente. Com o avanço da terapia clínica e das técnicas percutâneas, a revascularização do miocárdio é protelada em conseqüência disso.

Quanto as comorbidades associadas à cardiopatia isquêmica, achados semelhantes aos nossos foram encontrados em um estudo comparativo entre 1402 homens e 630 mulheres submetidos à RM isolada(9). No referido estudo, as taxas de HAS e hipercolesterolemia encontradas em homens e mulheres foram, respectivamente: $72,9 \%$; $88,9 \%$ e $55,1 \% ; 71,7 \%$. A pior condição clínica atual dos pacientes operados, representada pela maior prevalência de comorbidades como diabetes, HAS, dislipidemia, pode ser explicada pelo encaminhamento de pacientes com menor risco para a realização de angioplastia(6).

Quanto às variáveis cirúrgicas, $82,8 \%$ dos pacientes realizaram cirurgia de revascularização do miocárdio (CRM) isolada, a utilização de circulação extracorpórea (CEC) foi em 39,7\% das cirurgias. A mediana de tempo de permanência na UTI foi de 2,5 dias $(2,0-4,0)$. Os dados referentes às características cirúrgicas da população estudada estão demonstrados na Tabela 2.

Tabela 2: Características cirúrgicas da amostra $(n=58)$. Porto Alegre-RS, 2006.

\begin{tabular}{cc}
\hline Características & $\mathbf{n ~ ( \% )}$ \\
\hline CRM & $48(82,8)$ \\
CRM + cirurgia de válvula & $10(17,2)$ \\
No de vasos lesados (com TCE) & $2,6 \pm 0,7$ \\
Lesão de TCE & $17(29,3)$ \\
No de pontes realizadas & $3,4 \pm 1,1$ \\
Tempo cirurgia (horas) & $5,5 \pm 1,2$ \\
Uso de CEC (ambas as cirurgias) & $23(39,7)$ \\
Tempo CEC (minutos) & $84,9 \pm 30,2$ \\
Permanência na UTI (dias)* & $2,5(2,0-4,0)$ \\
\hline
\end{tabular}

* Dado apresentado com mediana e percentis interquartis. ${ }^{\dagger} n=23$. Demais variáveis contínuas apresentadas com média \pm desvio padrão. Dados categóricos apresentados com n (\%). CRM: cirurgia de revascularização do miocárdio, CEC: circulação extracorpórea, TCE: tronco da coronária esquerda.

Em relação ao tipo de cirurgia, a grande maioria dos pacientes submeteu-se à RM isolada e a utilização de CEC ocorreu em $39,6 \%$ das cirurgias. Embora a cirurgia realizada com CEC tenha sido considerada "padrão ouro" no tratamento da cardiopatia isquêmica multiarterial, seu uso tem sido bastante questionado devido aos seus danos(10).

A mediana de tempo de permanência na UTI, encontrada em nosso estudo, foi de 2,5 dias. Esse achado possivelmente possa ser explicado devido às rotinas de alta da UTI: pacientes submetidos à cirurgia de RM, que não apresentam nenhum tipo de complicação e se encontram em condições estáveis, têm alta no $2^{\circ}$ dia pós-operatório.

As complicações pós-operatórias ocorreram em 28 pacientes, o que representa $48,3 \%$ da amostra, sendo a mais prevalente a fibrilação atrial. Dados demonstrados na Gráfico 1. Do total de pacientes que apresentaram complicações, $5(8,6 \%)$ foram a óbito. As causas de óbitos foram: acidente vascular encefálico isquêmico $(3,5 \%)$, parada cardiorrespiratória $(1,7 \%)$, reoperação $(1,7 \%)$ e instabilidade hemodinâmica $(1,7 \%)$. 
Gráfico 1: Percentual de complicações pós-operatórias. Porto Alegre-RS, 2006.

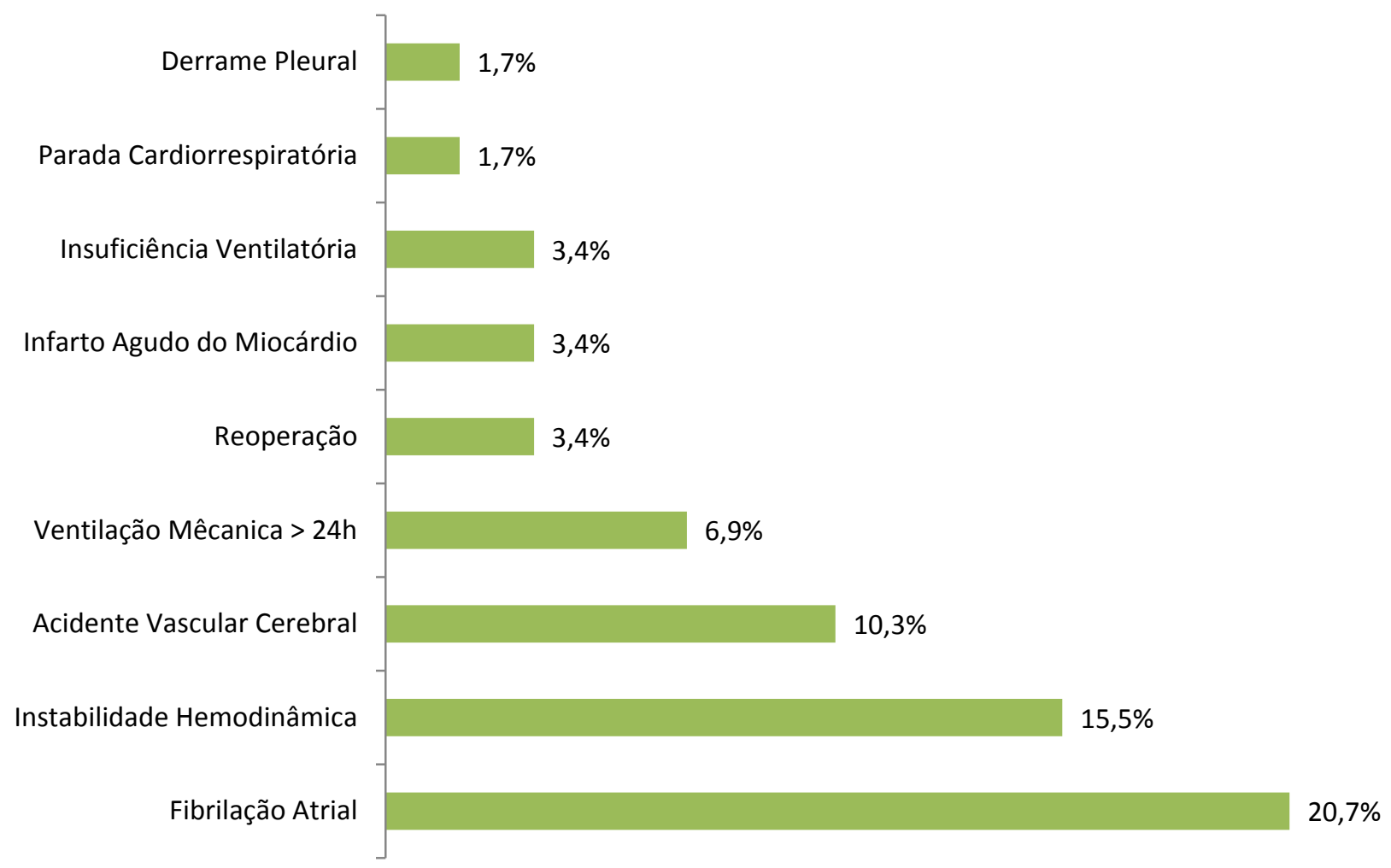

A fibrilação atrial foi a complicação mais frequente $(20,7 \%)$ nos pacientes deste estudo, dado semelhante ao encontrado na literatura(5). Essa arritmia atrial é a mais comum em cirurgia cardíaca. Ela pode surgir em $11 \%$ a $40 \%$ de pacientes depois de cirurgia de revascularização miocárdica e em aproximadamente $50 \%$ dos pacientes submetidos à cirurgia valvar ${ }^{(11)}$.

Dentre as causas de mortalidade pós-operatória na UTI, está o acidente vascular encefálico (AVE) isquêmico, responsável por 02 dos 05 óbitos em nossa amostra. O AVE está relacionado com a morbidade pós-operatória e é encontrado em 1 a $3 \%$ dos pacientes, geralmente associado à fibrilação atrial. Além disso, o IAM e a mediastinite também contribuem para a morbidade pós-operatória, sendo que a mediastinite está associada ao tempo de cirurgia, à presença de diabetes e ao uso da artéria mamária(3). A idade avançada, RM prévia, disfunção ventricular, cirurgia de emergência e comorbidades como insuficiência renal e lesão de tronco da coronária esquerda constituem fatores de risco para mortalidade hospitalar após a RM cirúrgica(3).

A instabilidade hemodinâmica, em nosso estudo, foi responsável por $15,5 \%$ das complicações. De acordo com dados da literatura(12), no período pósoperatório, o principal fator de risco para o desenvolvimento de disfunção renal é a instabilidade hemodinâmica, com sinais de baixo débito, cujos indicadores são hemorragia, uso de drogas inotrópicas e balão intra-aórtico. Tais evidências confirmam a necessidade da equipe de enfermagem, por ser a categoria profissional com maior grau de proximidade com o paciente, estar capacitada para avaliar constantemente os sinais clínicos de alterações hemodinâmicas, tais como perfusão periférica, débito urinário, drenagem pleural e de mediastino, sinais vitais, infusões endovenosas e assim, comunicar o médico intensivista com vistas a prevenir maiores complicações nesses indivíduos.

A ventilação mecânica prolongada foi outra complicação identificada, considerada um fator que pode provocar a ocorrência de infecção respiratória e influenciar no tempo de permanência do paciente na UTI. Um estudo(12) constatou que, quanto maior a sedação do paciente, ao ser admitido na unidade, mais rápido é retirado da ventilação mecânica. Esse achado foi relacionado com a ausência de dor sentida no pós-operatório de cirurgia cardíaca. Um paciente sem dor acorda mais tranqüilo da anestesia e colabora no processo de desmame. A dor acarreta limitação dos movimentos. Portanto, tosse, respiração profunda e mudanças de posição estarão restritas. Consequentemente, o paciente acordará agitado, ansioso, podendo ocorrer contenção mecânica no leito e sedação, prolongando assim, a saída da ventilação mecânica e extubação(12).

A análise da associação entre as características demográficas, clínicas e cirúrgicas da amostra e as complicações pós-operatórias mostrou que a idade e o tempo de permanência na UTI estiveram associados com a presença de complicações, 
conforme mostra a Tabela 3. Este dado denota que os indivíduos com maior idade tiveram mais complicações, assim como o tempo de permanência na UTI foi maior para indivíduos com complicações. Para as demais características não houve diferenças estatisticamente significativas. Para verificar se houve associação entre a idade e o tempo de permanência na UTI, foi realizada uma correlação, que demonstrou fraca associação entre essas variáveis $\left(r_{s}=0,318\right)$.

Tabela 3: Comparação entre o grupo de pacientes com e sem complicações pós-operatórias em relação às características da amostra. Porto Alegre-RS, 2006.

\begin{tabular}{|c|c|c|c|}
\hline \multirow{2}{*}{ Características } & \multicolumn{2}{|c|}{ Complicações n (\%) } & \multirow{2}{*}{$\mathbf{P}$} \\
\hline & $\operatorname{Sim}(n=28)$ & Não $(n=30)$ & \\
\hline Sexo masculino & $20(71,4)$ & $21(70,0)$ & 0,99 \\
\hline Lesão TCE & $8(28,6)$ & $9(30)$ & 0,99 \\
\hline Cirurgia de válvula associada & $4(14,3)$ & $6(20)$ & 0,49 \\
\hline Uso de CEC & $11(36,7)$ & $12(42,9)$ & 0,79 \\
\hline HAS & $26(86,7)$ & $24(85,7)$ & 0,99 \\
\hline DM & $9(30,0)$ & $12(42,9)$ & 0,41 \\
\hline IMC & $29,1 \pm 4,7$ & $28,7 \pm 3,6$ & 0,67 \\
\hline Idade & $68,1 \pm 9,2$ & $62,8 \pm 9,7$ & 0,038 \\
\hline FE (\%) & $55,2 \pm 17,2$ & $64,9 \pm 21,5$ & 0,065 \\
\hline Permanência na UTI* (dias) & $4,0(3,0-5,75)$ & $2,0(2,0-2,0)$ & $<0,001$ \\
\hline
\end{tabular}

* Dado apresentado com mediana e percentis interquartis; demais dados contínuos apresentados com média \pm desvio padrão. TCE: tronco de coronária esquerda, CEC: circulação extracorpórea, HAS: hipertensão arterial sistêmica, DM: diabetes mellitus, IMC: índice de massa corporal, FE: fração de ejeção.

Nosso estudo demonstrou que pacientes mais idosos apresentaram mais complicações, o que os levaram a permanecer por mais tempo na UTI. Frequentemente são os pacientes mais idosos que sobreviveram a um infarto ou já realizaram intervenção percutânea, apresentando maiores riscos de complicações trans e pós-operatórias(11). Em consequência disso, o período pós-operatório, principalmente nas primeiras 48 horas após cirurgia cardíaca, exige cuidados intensivos e especializados a fim de se detectar precocemente alterações de todos os tipos e evitar seus efeitos deletérios.

Neste contexto, entre as complicações identificadas em nosso estudo e, de acordo com a literatura(13-14), torna-se fundamental a identificação precoce de alterações no ritmo cardíaco, a administração de medicações tão logo sejam prescritas, seja para controle do ritmo, da instabilidade hemodinâmica ou da dor, o acompanhamento no processo de desmame da ventilação mecânica, além da adequada utilização das medidas de precaução padrão e técnicas assépticas para realização de procedimentos, prevenindo infecções. Novos eventos tornam a permanência do paciente prolongada na UTI, gerando custos crescentes.

\section{Implicações para a Prática}

Sabe-se que a falta de habilidade e atenção é fator determinante para a ocorrência de falhas durante a assistência de enfermagem a pacientes em recuperação pós-operatória imediata. O treinamento deficiente, a observação assistemática e a indefinição de papéis na equipe de enfermagem constituem as principais causas de falhas na assistência(15-16). Diante da modificação do perfil clínico dos pacientes submetidos à cirurgia cardíaca e às complicações mais frequentes, torna-se fundamental a qualificação da assistência de enfermagem, visando à diminuição da morbimortalidade. Nesse contexto, os objetivos poderão ser alcançados a partir de ações essenciais como treinamentos, definição de papéis dentro da equipe, reestruturação física, além da sistematização da assistência de enfermagem para o recebimento de pacientes no pós-operatório imediato, quando as atividades, sequencialmente especificadas por um enfermeiro, sejam desenvolvidas para garantir o melhor cuidado possível.

\section{CONCLUSÃO}

Os dados levantados estão de acordo com a literatura, que menciona pacientes homens, mais velhos e com maior prevalência de comorbidades, como HAS, dislipidemia, diabetes e sobrepeso ou obesidade. A maior gravidade dos pacientes é confirmada pelo histórico clínico mencionado. Há uma redução na freqüência de uso de CEC na cirurgia cardíaca de RM e a fibrilação atrial continua sendo a complicação pós-operatória mais frequente. O conhecimento acerca do perfil clínico e cirúrgico dos pacientes operados proporciona subsídios para a equipe de enfermagem planejar e garantir o cuidado básico e avançado em UTI, frente às condições adversas que os pacientes poderão apresentar.

\section{REFERÊNCIAS}

1. MCGovern PG, Pankow JS, Sahar E, Doliszny KM, Folsam AR, Blackburn $H$, et al. Recent trends in acute 
coronary heart disease: mortality, morbidity, medical care and risk factors. $\mathrm{N}$ Engl J Med. 1996;334(14):884-90.

2. Behr PE. O sexo feminino como fator de risco para a mortalidade hospitalar após a cirurgia de revascularização miocárdica [dissertation] Porto Alegre: Fundação Universitária de Cardiologia; 2001.

3. Sociedade Brasileira de Cardiologia. Diretrizes da cirurgia de revascularização miocárdica. Arq Bras Cardiol. 2004;82 Supl 5:1-20.

4. Almeida FF, Sandhi MB, Couto BR, Starling CE. Fatores preditores da mortalidade hospitalar e de complicações pré-operatórias graves em cirurgia de revascularização do miocárdio. Arq Bras Cardiol. 2003;80(1):41-50.

5. Warner CD, Weintraub WS, Craver JM, Jones EL, Got JP, Guyton RA. Effect of cardiac surgery patient characteristics on pacient outcomes from 1981 througt 1995. Circulation. 1997;96(5):1575-79.

6. Feier FH, Sant'anna RT, Garcia E, De Bacco F, Pereira E, Santos MF, et al. Modificações no perfil do paciente submetido à operação de revascularização do miocárdio. Rev Bras Cir Cardiovasc. $2005 ; 20(3): 317-22$.

7. Carvalho ARS, Matsuda LM, Carvalho MSS, Almeida RMSSA, Schneider DSLG. Pós-operatório de revascularização miocárdica: tipos de complicações. Ciênc. cuid. saúde. 2006;5(1):50-9

8.Irmandade Santa Casa de Misericórdia de Porto Alegre. Relatório anual de dados gerenciais. Porto Alegre: Irmandade Santa Casa de Misericórdia; 2004. 9. Amato VL, Timerman A, Paes AT, Baltar VT, Farsky PS, Farron JA, et al. Resultados imediatos da cirurgia de revascularização miocárdica: comparação entre homens e mulheres. Arq Bras Cardiol. 2004;83 [spe]: 14-20.

10. Pinheiro BB, Fagundes WV, Ramos MC, Azevedo VLB, Silva JM. Revascularização do miocárdio sem circulação extracorpórea em pacientes multiarteriais: experiência de 250 casos. Rev Bras Cir Cardiovasc 2002;17(3):242-47.

11. Ambrov D, Tamariz MG, Fremes SE, Guru V, Borger MA, Christakis GT, et al. Trends in coronary artery bypass surgery results: a recent 9 years study. The Annal of Thoracic surgery. 2000;70(1):84-90.

12. Braz MR, Stipp MAC. Desmame ventilatório no pós-operatório de cirurgia cardíaca: um ensaio clínico. Online Braz J Nurs [Internet]. 2007 [cited dez 30];6(3). Available from: http://www.uff.br/objnursing/index.php/nursing/artic le/view/j.1676-4285.2007.1153/270.

13. Tineli RA, Rosa E Silva Junior R, Luciano PM, Rodrigues AJ, Vicente WVA, Evora PRB. Fibrilação atrial e cirurgia cardíaca: uma história sem fim e sempre controversa. Braz J Cardiovasc Surg. 2005;20(3):323-31.

14. Mangano CM, Diamondstone LS, Ramsay JG,
Aggarwal A, Herskowitz A, Mangano DT. Renal dysfunction after myocardial revascularization: risk factors, adverse outcomes, and hospital resource utilization. The Multicenter Study of Perioperative Ischemia Research Group. Ann Intern Med. 1998;128(3):194-203.

15. Chianca TCM. Falhas de enfermagem no pósoperatório imediato de pacientes cirúrgicos. Rev Latino-am Enfermagem. 2006;14(6):879-86.

16. Jorgetto GV, Noronha R, Araújo IE. Assistência de enfermagem a pacientes cirúrgicos: avaliação comparativa. Rev. Eletr. Enf. [Internet]. 2005 [cited 2009 dez 30];7(3):273-7. Available from: http://www.fen.ufg.br/Revista/revista7 3/original 03.htm.

Artigo recebido em 28.11.08.

Aprovado para publicação em 14.09.09.

Artigo publicado em 31.12.09. 